\title{
An Improved Method for Decreasing Intermediate Density Elements in Topology Optimization
}

\author{
Zaizhuo Jianga ${ }^{\mathrm{a}}$, Li Zhang ${ }^{\mathrm{b},}{ }^{*}$, Yingbing Wan ${ }^{\mathrm{c}}$ and Cong Jin ${ }^{\mathrm{d}}$ \\ School of Mechanical Science and Engineering, Huazhong University of Science \& Technology, \\ Wuhan 430000, China \\ aM201570296@hust.edu.cn, bzImse@mail.hust.edu.cn
}

Keywords: Topology optimization, Density gradient message, SIMP, OC.

\begin{abstract}
The instability phenomenon of numerical solution usually exists in topology optimization of continuum structure. The sensitivity filter method is used to solve this instability phenomenon of numerical solution generally, but many intermediate density elements still exist in the result of topology optimization. Intermediate density elements are difficult to manufacture in the industry, so it is urgent to form an efficient method to decrease them. This paper proposes a improved method-considering density gradient message and elements numbers in the filter radius. It sets up a model which focus on modifying the weighting function and the elements' density within the filter radius. It is based on Solid Isotropic Material with Penalization (SIMP). The optimality criteria method (OC) is used to solve this model. Finally a widely studied example is used to demonstrate the availability and advantage of the proposed filter method.
\end{abstract}

\section{Introduction}

Topology optimization can be defined as a mathematical method that optimizes material layout within a given design space, for a set of given loads, boundary conditions and constraints with the goal of maxing the performance of the system. There are many methods in structure topology optimization area, but any method has limitations. Checkboard phenomenon and mesh-dependency always appear in SIMP [1] method. These problems make it difficult to manufacture the structure. So many methods have emerged in this area such as higher order element and nonconforming element method [2], perimeter control method [3], local gradient method [4] and filter method. Filter method contains sensitivity filter method [5] and density filter method [6]. Weisheng Zhang [7] introduced an improved weighting mechanism proposes in the topology optimization process, Kai Long [8] proposed density gradient to filter the sensitivity. Xiaojia Zhang [9] proposed a discrete filtering scheme. These methods can not eliminate the intermediate density elements. In this paper an improved method is proposed to decrease the munber of intermediate density elements.

\section{Numerical Model}

The topology optimization method of continuum structure began in 1988 by Bendoe's homogenization method [10]. On this basis, it evolved into the most popular variable density method. The difference between the variable density method and the homogenization method is that there is no need to introduce the microstructure model, but the design domain is assumed to be an artificial density unit. The usual practice is that the elastic modulus between the material in the design domain and the relative density of the element through the interpolation method to link up. There are two interpolation methods SIMP and RAMP. Here is the SIMP numerical model

$$
\begin{aligned}
& E\left(x_{i}\right)=E_{\text {min }}+\Delta E x_{i}^{p} \quad \Delta E=E-E_{\text {min }}, i=1,2, \ldots, n e \\
& \mathbf{K}=\sum_{i=1}^{n e}\left(E_{\text {min }}+\Delta E x_{i}^{p}\right) \mathbf{K}_{i}
\end{aligned}
$$

The standard notion for minimum compliance design problems can be mathematically defined as follow: 


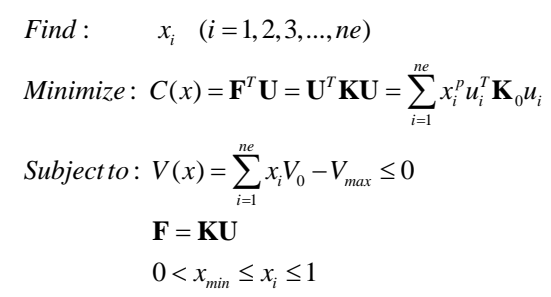

Where the equality constraint is introduced to limit the material usage, and $V_{\max }$ is the prescribed volume. $V_{0}$ is the volume when the element's relative density is one. $\mathbf{F}$ is the external load, $\mathbf{U}$ is the displacement field, $X_{i}$ is the design variable, $n e$ is the number of elements and $x_{\min }=0.001$.

Methods for solving topology optimization are mathematical programming (MP), Artificial Intelligence Optimization Algorithm (AIOA), optimality criteria (OC). The OC algorithm does not directly optimize the objective function as a mathematical programming method, which attempts to satisfy a series of guidelines related to structural behavior in an indirect way, either derived from design experience or from rationality, development has gone through a process from empirical criteria to rational law. The empirical criterion method is kind of the criteria established in the early stage of structural optimization, such as equal strength design criterion, synchronization failure criterion, full stress design criterion and full strain energy criterion. The updating of the variables in the OC algorithm is based on some explicit heuristic iterative formulas constructed by the criterion. The formula often introduces some empirical coefficients to adjust the convergence and stability of the optimization process, such as the step size factor, damping factor and other factors. The updating scheme can be shown as follow:

$$
x_{i}^{(k+1)}=\frac{\sum_{i=1}^{n e} p x_{i}^{p-1} u_{i}^{T} k_{0} u_{i}}{\lambda_{1} \sum_{i=1}^{n} v_{i}} x_{i}^{(k)}
$$

The termination condition of the optimization iteration is $\left|x_{i}^{(k+1)}-x_{i}^{(k)}\right| \leq \varepsilon$, where $\varepsilon$ is a very small number 0.001 .

\section{The original filter method and improved method}

\subsection{Original filter method}

Sensitivity filter is always carried out as this two steps: Calculate the sensitivity of each design variable $\frac{\partial f}{\partial x_{i}}$ firstly then update the sensitivity by weight function. The weight function is as follow:

$$
H_{i}=R-\operatorname{dist}(i, k),\{i \in N \mid \operatorname{dist} \leq R, k=1,2, \ldots N\}
$$

Where $\mathrm{R}$ and $\mathrm{N}$ are filter radius and the numbers of elements within the filter radius respectively. $\operatorname{dist}(i, k)$ represents the distance of element $\mathrm{i}$ and element $\mathrm{k}$. This filter scheme can be defined as:

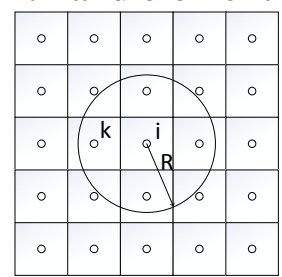

Fig. 1 Filter Scheme

Sensitivity filter formula is defined by Sigmund. In order to obtain clearer topology optimization boundary, Borrvall [11] proposed a modified sensitivity filter formula. In the topology optimization of multi-physics fields [12], the density in the formula is always leave out. Through the above three methods of numerical experiments we can see there still many intermediate density elements in the topology optimization structure. So this paper proposes a method considering density gradient message and elements numbers in the filter radius in topology optimization. 


\subsection{Improved method}

The proposed method considers density gradient message and elements numbers in the filter radius, the former focus on modifying the weighting function and the later focus on modifying the elements' density within the filter radius. So the new sensitivity can be presented as follow:

$$
\begin{aligned}
& \frac{\partial \hat{f}}{\partial x_{i}}=\frac{\sum_{i \in N} \hat{H}_{i} \cdot H_{i} \cdot x_{i} \frac{\partial f}{\partial x_{i}}}{\left[\left(x_{k}\right)^{n}+\left(\sum_{j=1}^{4} x_{j}\right) / 4\right] \cdot \sum_{i \in N} \hat{H}_{i} \cdot H_{i}} \\
& \text { Where } \hat{H}_{i}= \begin{cases}1 & \left|x_{i}-x_{k}\right| \leq \alpha \\
0.001 & \left|x_{i}-x_{k}\right|>\alpha\end{cases}
\end{aligned}
$$

This improved method on the one hand identifies the elements on the boundary of the structure and modify the weighting function to decrease intermediate density of the structure boundary, on the other hand modifying the elements' density within the filter radius by controlling the number of elements. The parameters are set as $\eta=0.5, \alpha=0.65$, we chose the average of the biggest four elements' density as the element's density. We chose the parameter $x$ to present the number of intermediate elements, the smaller $x$ the less number of intermediate elements and the filter effect is better.

$$
\bar{x}=\sum_{i=1}^{n e} x_{i} \cdot\left(1-x_{i}\right)
$$

\section{Case study}

This part is based on MATLAB2012 environment. The design area is discrete by Plane four-node rectangular element, elements are all unit, the elastic modulus is 1 and Poisson's ratio is 0.3 and penalty factor is 3 .

2D Cantilever beam design area is as follow, Aspect ratio of the area is $2: 1$, the external load is $1 \mathrm{kN}$. The objective function is compliance with $50 \%$ volume fraction. This design area is meshed by $100 \times 50$ whose filter radius is 1.5 .

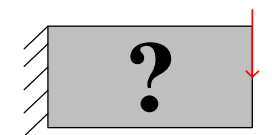

Fig. 2 Design Area

Table 1 Comparing of two methods

\begin{tabular}{ccc}
\hline Method & compliance & $\bar{x}$ \\
Original filter method & 72.4632 & \\
Improved method & 66.7489 & \\
\hline
\end{tabular}

Original filter method got the objection compliance value $72.4632, \bar{x}=165.8916$. While improved method that we proposed got the objection compliance value $66.7489, x=3.9020$. We can see that improved method result is better than original filter method by comparing compliance and $\bar{x}$. At the same time no thin structures exist in the topology optimization result. That is good for manufacturing.

\section{Conclusion}

In this paper, an improved method is proposed to update the original sensitivity filtering function by considering the density gradient information and modifying the elements' density within the filter radius. The improved method shows the advancement of reducing the number of intermediate density elements. The phenomenon of checkerboard in numerical calculation is solved. However, there are still some shortcomings of the filter method proposed in this paper, the number of intermediate 
density elements decreased obviously, but it makes the structure of the boundary appears jagged, the problem will be solved in the future research.

\section{Acknowledgments}

This work was financially supported by National Natural Science Foundation of China (Grant No:51405170) and The National High-Tech Research and Decelopment Program (863Program)of China 2015AA042505.

\section{References}

[1] Sigmund O. Design of material structures using topology optimization, $\mathrm{PhD}$ Dissertation, Technical University of Denmark Academic, 1994

[2] Jang G W, Jeong J H, Kim Y Y, et al. Checkerboard-free topology optimization using non-conforming finite elements [J]. International Journal for Numerical Methods in Engineeriing, 2003, 57(12): 1717-1735

[3] Haber R B, Jog C S, Bendsoe M P.A new approach to variable-topology shape design using a constraint on perimeter [J]. Structural Optimization, 1996, 11(1/2): 1-12

[4] Petersson J, Sigmund O. slope constrained topology optimization[J].International Journal for Numerical Methods in Engineering, 1998, 41(8): 1417-1434

[5] Sigmund O.A 99 lines topology optimization cade written in MATLAB [J]. Structural and Multidisciplinary Optimization. 2001, 21(2): 120-127

[6] Bourdin B. Filters in topology optimization [J]. International Journal for Numerical Methods in Engineering. 2001, 50: 2143-2158

[7] Weisheng Zhang. Studies on Stress-related topology optimization problem via level set approach [D]. Dalian: Dalian University of Technology, 2013

[8] Kai Long. Sensitivity filtering method considering density gradient [J]. Journal of computer-aided design \& computer graphics. 2015, 26: 669-674

[9] Xiaojia Zhang. Material nonlinear topology optimization using the ground structure method with a discrete filtering scheme [J]. Structural and Multidisciplinary Optimization.2017, 52(6): 1032-1060

[10] Bendsoe M.P., Kikuchi N., Generating optimal topologies in structural design using a homogenization method [J]. Computer Methods in Applied Mechanics and Engineering. 1988, 71: $197-224$

[11] Borrvall T. Topology optimization of elastic continua using restriction [J]. Archives of computational methods in engineering.2001,8(4): 351-385

[12] Sigmund O. Design of multi-physics actuators using topology optimization - part II: two material structures [J]. Computer Methods in Applied Mechanics and Engineering, 2001, 190(49/50): 6605-6627 